\title{
BIOLOGIA REPRODUTIVA DE JUMENTOS. III. pH, OSMOLALIDADE E NÍVEIS DE ELETRÓLITOS NO SEMEN*
}

\author{
REPRODUCTIVE BIOLOGY OF DONKEY. III. PH, OSMOLALITY AND ELETROLYTES CONCENTRATION OF THE \\ SEMEN
}

Rosana Nogueira de MORAIS'; Raul Gastão MUCCIOLO ${ }^{2}$; Wilson Gonçalves VIANA ${ }^{3}$

\begin{abstract}
RESUMO
Amostras de sêmen e plasma seminal de seis jumentos da raça Pêga foram analisadas para determinação do $\mathrm{pH}(\mathrm{n}=70)$, osmolalidade $(n=69)$ e níveis de $\mathrm{Na}(\mathrm{n}=49), \mathrm{K}(\mathrm{n}=51), \mathrm{Ca}(\mathrm{n}=51)$ e $\mathrm{Mg}(\mathrm{n}=51)$. Os valores de $\mathrm{pH}$ variaram de 7,0 a $7,8 \mathrm{com}$ uma média de $7,29 \pm 0,18$ para o total de cjaculados. A osmolalidade do plasma seminal oscilou entre 273 e $373 \mathrm{mOsm}$ tendo como média global o valor de $302,89 \pm 15,03 \mathrm{mOsm}$, o que corresponde a um ponto crioscópico de $-0.56^{\circ} \mathrm{C}$. As concentrações média de $\mathrm{Na}, \mathrm{K}$. Ca e $\mathrm{Mg}$ foram, respectivamente, $238,26 \pm 67,92$. $105,20 \pm 32,14,13,24 \pm 1,62$ e $1,54 \pm 0,10 \mathrm{mg}$ para cada $100 \mathrm{ml}$ de plasma seminal. Os resultados obtidos no presente estudo foram confrontados com dados publicados relativos a jumentos e garanhōes, sendo em geral muito próximos. A exceção foram os níveis de $\mathrm{Mg}$ que se mostraram relativamente baixos nos animais por nós encaminhados. Pela semelhança existente entre as características seminais de asininos e eqüinos, acredita-se que os métodos utilizados para a congelaçāo de sêmen eqüino possam, em princípio, ser utilizados para o sêmen de jumentos. A confirmação desta hipótese fica na dependência de estudos complementares.
\end{abstract}

UNITERMOS: Jumentos; Reprodução; Sêmen; pH; Pressão osmótica; Eletrólitos

\section{INTRODUÇÃO E LITERATURA}

O efeito deletério do plasma seminal na conservação do sêmen equiino "in vitro" é reconhecido e descrito por vários autores 2, 5, 15, 20, 27, 32. Deste modo, para a congelação de sêmen usa-se separar o plasma seminal e substituí-lo por um diluente que seja compatível com as células espermáticas e, ao mesmo tempo, livre de efeitos tóxicos. No entanto, para a definição de um diluidor ideal torna-se necessário o conhecimento das características fisiológicas do plasma seminal a fim de conservar seus fatores benéficos. Nestes aspectos incluem-se muitas variáveis, dentre as quais algumas determinantes como o $\mathrm{pH}$, osmolalidade e níveis de eletrólitos.

A concentração de ions hidrogênio $(\mathrm{pH})$ no sêmen é, indubitavelmente, um dos fatores que mais afetam a motilidade, viabilidade e metabolismo dos espermatozóides nas várias espécies ${ }^{6,14.28}$. O pH ácido inibe o metabolismo, enquanto o alcalino, ao contrário, o estimula. De modo geral, as amostras de sêmen tendem a neutralidade, apesar dos espermatozóides de mamíferos apresentarem grande resistência às variações hidrogeniônicas ${ }^{14}$. A excessiva alcalinidade inicial do sêmen pode estar associada à baixa fertilidade do reprodutor, indicando, muitas vezes, baixa concentração

\footnotetext{
1 - Professor Assistente - Universidade Federal do Paraná

2 - Professor Associado - Faculdade de Medicina Veterinária e Zootecnia da USP

3. Professor Doutor - Faculdade de Medicina Veterinária e Zootecnia da USP
}

espermática ou um quadro de contaminação bacteriana do ejaculado 5,28 .

A faixa de variação de $\mathrm{pH}$ mais comumente encontrada para o sêmen de garanhões está entre 6,2 e 7,8 , com prevalência de $\mathrm{pH}=7,5^{18} \cdot 23.24 .27 .31$, sendo um parâmetro relativamente constante. Com base nesse conhecimento, o pH dos diluidores utilizados na conservação de sêmen eqüino deveria ser ajustado, através de diferentes substâncias tampão, para a faixa de 6,9 a 7,2 5 . Para jumentos prevalecem basicamente os mesmos valores encontrados para garanhões, sendo também uma característica seminal bastante constante. As médias obtidas por diversos autores oscilam entre $\mathrm{pH} 7,0 \mathrm{pH}$ $7,7^{4}, 7,8,9,11,19$.

Outro fator de suma importância para obtenção de bons resultados a partir das técnicas de preservação de sêmen é o conhecimento da osmolalidade do plasma seminal, uma vez que os espermatozóides sobrevivem melhor quando diluídos em soluçōes fisiológicas com osmolalidade próxima à do sêmen ${ }^{28}$.

- Projeto financiado pela FAPESP - Fundaçāo de Amparo à Pesquisa do Listado de São Paulo. 


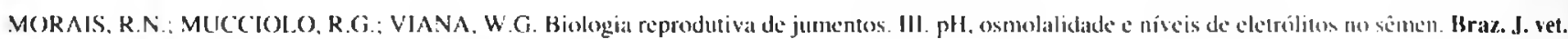
Res. anim. Scio., Sào Paulo, v. 31. n.2, p. 14.5-51. 1994.

A determinação da osmolalidade de um fluido biológico pode ser realizada através de várias técnicas. porém, a medida do ponto de congelamento (crioscopia) é a técnica de eleição para a maioria dos autores devido à sua simplicidade de execução e repetibilidade e precisão de resultados ${ }^{14.28 .33}$.

Fim garanhões. os resultados obtidos são bastante semelhanles entre si, variando desde $290 \mathrm{mOsm}$ até $33.3 \mathrm{mO}$ sm, o que corresponde a pontos crioscópicos entre $-\left(0,50^{\circ} \mathrm{C}\right.$ e $-\left(0,62^{\circ} \mathrm{C}^{18}\right.$ $24,26,27,30.44$. enquanto que em jumentos variam de 301 a 3.3 .3 $\mathrm{mOsm}$, correspondendo a pontos crioscópicos de $-0,56^{\circ} \mathrm{C}$ e $-0,62^{\circ} \mathrm{C}$. respectivamente ${ }^{14}$. Com base nestes dados, acredita se que a pressāo osmótica de um diluidor para a congelação do sêmen de jumento deve ser próxima ou ligeiramento supcrior à do plasma seminal. ou seja, entre 300 e 330 InOsm20.27.

Um terceiro aspecto importante a ser avaliado são os niveis de eletrólitos presentes no plasma seminal. De modo geral. cátions mais abundantess no sêmen das espécies domésticăs são o sódio ( $N_{a}$ ) e o potássio $(K)$. com menores concentraçiò de cálcio (Ca) e magnésio (Mg). A função desses ions estai relacionada basicamente com a mamutenção do equilíbrio osmótico e motilidade espermática ${ }^{1+2}$. 0 Na c o K são os mais importantes na manutençāo da pressão osmótica e. normalmente, apresentam correlação negativa entre si, sendo difícil estabelecer-se a funçāo de um. independente do outro.

Ao se avaliarem os efeitos. em conjunto, do $\mathrm{pH}$, da pressão osmótica e dos níveis de Ca e K sobre o metabolismo aeróbico de espermatomóides hovinos, as menores taxas de atividade. dadas pelo indice frutolítico e produção de ácidos láticos, coincidiram com os valores mais baixos de $\mathrm{pH}$ e de pressão osmólica e altos níveis de K. O Ca nāo apresentou nenhum efeito marcante sobre o metabolismo espermático, mas eliminou virtualmente todos os efeitos inibitórios dos elevados níveis de $\mathrm{K}^{6}$. O fluido epididimário, comparativamente ao plasma seminal, apresenta proporção $\mathrm{N} a \mathrm{~K}$ bastante baixa. em decorrência de altas concentrações de $\mathrm{K}$. Tal fato atua como inibidor natural da atividade metabólica excessiva dos espermatozóides, determinando um estado de quiescência celular, que, permite maior tempo de sobrevivência para as células espermáticas durante sua passagem pelo epidídimo. $\mathrm{O}$ aumento da proporção $\mathrm{Na} / \mathrm{K}$, juntamente com a inibição do $\mathrm{K}$ pelo Ca, à nível de ejaculado estão implicados com aquisição de motilidade pelos espermatozóides ${ }^{14}$

Em eqüinos, os níveis de $\mathrm{Na}, \mathrm{K}, \mathrm{Ca}$ e $\mathrm{Cl}$ contribuem em $92 \%$ para a pressão osmótica do sêmen e níveis elevados de Na sāo prejudiciais à conservação do ejaculado ${ }^{12}$. Com base nestes dados avaliou-se o efeito da variação da taxa $\mathrm{Na} / \mathrm{K}$ no diluidor HS2 $\mathrm{O}^{17}$ sobre a conservação de espermatozóides equïinos resfriados ou congelados. obtendo-se melhora de 4 a $7 \%$ na motilidade quando a proporção Na/K foi reduzida de 10.32 para 3,5. Concluiu-se que os diluentes muito ricos em $\mathrm{Na}$ poderiam levar a um esgotamento das reservas enzimáticas ou energéticas. ou ainda. a uma entrada massiva de fons $\mathrm{Na}$ na cćlula, destruindo seu equilíbrio iônico's

A concentração dos principais íns presentes no plasma seminal de garanhões foi determinada por diversos autores. Os níveis de Nal variam de 220 a 28.5 mgr/ 1. 12, 14. 21, 29, 34. sendo relativamente constantes. Com relação alos demais eletrólitos. existe maior variabilidade entre os resultados.

A concentraçāo de $\mathrm{K}$ varia desde $5.5 \mathrm{mg} / \mathrm{ate} 10.3 \mathrm{mg} \%$. e a le Ca de 9.74 alé $29,3 \mathrm{mg} \%$. 12. 2. i. () íon de menor concentração entre aqueles estudados a $\mathrm{Mg}$. que está presente na quantidade de 4.2 até $9 \mathrm{mg}$ em cada $100 \mathrm{ml}$ de plasma seminal ${ }^{16,21,29,3.4}$.

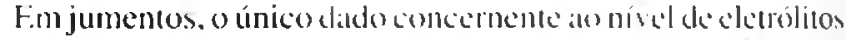
no plasma seminal foi aprenéntado por MINN el all. "(1963). com uma média de $74.3 .5 \mathrm{mg} \%$ para a concentração de $\mathrm{NaCl}$. o que equivale, aproximadamente. a niveis de $242 \mathrm{mg} \% \mathrm{de}$ Na. no mesmo material.

\section{MATFRIAI, F, METODO}

\section{ANIMAIS}

Foram utilizados seis (6) jumentos da raça lềa. pertencerites a três propriedades rurais do Município de São (arlos - SP, os quais foram designados por letras de $A$ a 1 . A altura e peso médios foram de $1.30 \mathrm{~m}$ e $400 \mathrm{~kg}$. respectivamente. e a idade de cada um no início do experimento era de + (A), 6 (B), 6 (C). 8 (D), 9 (F) e 3 (F) anos. Todos foram submetidos previamente a exame clínico e andrológico, tendo sido considerados aptos para o experimento. Durante lodo o período experimental, de outubro de 1988 a abril de 1989. os animais foram mantidos em piquetes individuais. com livre acesso a baias. recebendo ração c suplementação mineral balanceadas, com água à vontades.

\section{COLHEITA DE SÊMEN}

A técnica de colheita foi rualizada de acordo com PICKETT et al. ${ }^{25}$ (1987), sendo utilizada vagina artificial modelo tipo Colorado. Como manequim foram utilizadas éguas em cio natural e, apenas para o jumento $C$, foram utilizadas jumentats em cio por se tratar de um reprodutor nào habituado a realizar coberturas em éguas.

Após a colheita, o sêmen foi levado incdialamente ao laboratório para ser analisado. 
MORAIS. R.N.: MUC(IOI.O. R.G.; VIANA, W.(i. Biologia reprodutiva de jumentos. III. pH, osmolalidade a niveis de aletrólitos no sêmen. Braz. J. vet. Res. anim. Sci.. São Paulo, v. 31. n.2, p. 145-51. 1994.

\section{PH DO SE.MEN}

OpH decada cjaculado foi decerminado at ravés do pHmetro** com eletrodo de vidro combinado****, devidamente calibra-

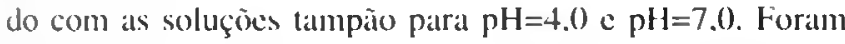
feitas duas leituras de cada amostra e registrada a média das mesmas.

\section{OSMOLALIDAIDF: DO PLASMA SEMINAI.}

Lima alíquota de sêmen foi centrifugada a $1800 \mathrm{x}$ g durante 15 minutos para a obtenção do plasma seminal a ser utilizado na determinação da osmolalidade $(5$ a $6 \mathrm{ml}$ ). As amostras. obtidas em duplicata. foram devidamente identificadas e mantidas a $-20^{\circ} \mathrm{C}$ até serem analisadas.

A osmolalidade foi determinada através da medida do ponto de congelamento de cada amostra em crioscópio eletrônico digital*k**. l'ara se chegar ao valor da osmolalidade (O) expressa em mOsm. partiu-se do princípio que 1 mol de uma substância não eletrolítica, adicionada a $1 \mathrm{~kg}$ de água (solução) molal) abaixa o ponto de congelamento $\mathrm{cm} 1,86^{\circ} \mathrm{C}$ (constante de depressão do ponto de congelamento molal $)^{33}$. Deste modo utilizou-se a seguinte equação :

$0=\Delta / 1.86 \times 10^{4} \mathrm{mOsm}$. onde $\Delta=$ ponto crioscópico da amostra em ${ }^{\circ} \mathrm{C}$

\section{NÍVEIS IDE I:L.ETRÓI.ITOS NO PL.ASMA SEMINAL} As concentrações de sódio (Na), potássio (K). cálcio (Ca) e magnésio ( $\mathrm{Mg}$ ) foram determinadas nas duplicatas disponíveis da amostra de plasma seminal obtidas para avaliação da

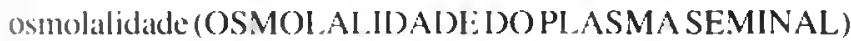
para o Ca, K e Mg. foram analisadas 51 amostras, enquanto que para o Na. apenas 49 amostras.

As dosagens de Na, Ca e $\mathrm{K}$ foram realizadas através de espectrofotometria de emissão atomica*****. sendo que para o Va foi utilizado o método de adição de padrão. enquanto que para o $\mathrm{K}$ e Ca seguiram-se os princípios básicos da técnica ${ }^{3}$ com uso de soluçōes padronizadas. Já para o $\mathrm{Mg}$ as dosagens foram feitas em espectrofotometro de absorção atômica******, seguindo as normas de uso do aparelho.

ANÁLISE I:STATIŚSTICA - Foram efetuados os cálculos da média aritmélica, desvio padrão e coeficiente de variação dos resultados obtidos para cada variável estudada, por reprodutor e para o lotal de amostras.

Para o estudo da correlaçāo entre as variáveis foram calculados os coeficientes de correlação de Pearson e os níveis de significância dos mesmos, utilizando-se o pacote estatístico SPSS-PC+*******.

*. $\mathrm{PH}$-meter E-520 - METROHM

** MICRONAL

*** Crioscópio 312 L I.AKTRON - Londrina - PR

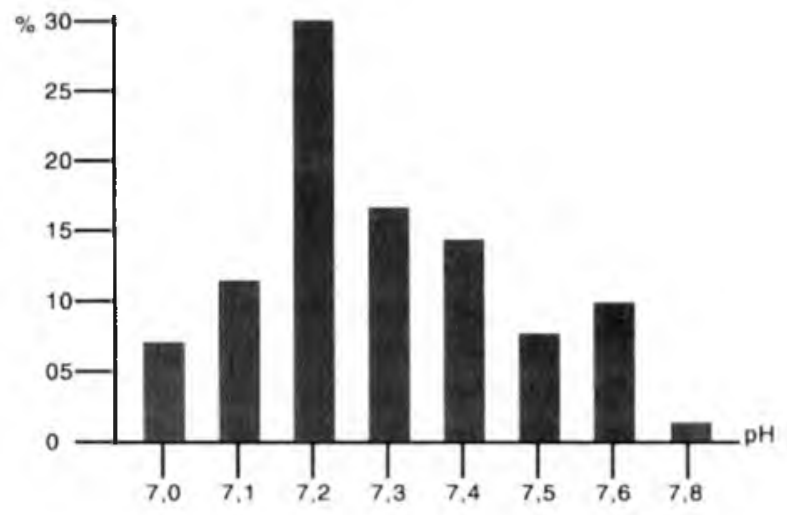

FICILRA

Porcentagem de exorrência dos valores de pll no ejaculado de jumentes $(n=70)$. Säo Carlos - SP. $1988 / 89)$

\section{RESUITADOS}

\section{$\mathrm{pH}$}

As médias obtidas para o $\mathrm{pH}$ dos ejaculados, tanto global como para cada reprodutor, estāo demonstradas na Tab.l, incluindo os valores máximos e mínimos. A diferença entre as amostras foi muito pequena. com um coeficiente de variação de apenass $2 \%$. O valor 7.2 foi o que ocorreu com maior frequêencia $(30 \%)$ como se pode observar na Fig. 1.

\section{OSMOLAI.IDADE:}

A média obtida para o ponto crioscópico das 69 amostras analisadas foi $-0.562 \pm 0.028^{\circ} \mathrm{C}$, variando de $-\left(0.5\left(17\right.\right.$ a $-\left(0,694^{\circ} \mathrm{C}\right.$. $O$ coeficiente de variação entre as amostras foi bastante baixo $(4 \%)$.

Na Tab. 2 encontram-se os valores médios da osmolalidade do plasma seminal para cada reprodutor e para o total de amostras analisadas.

\section{NÍVFIS IDE: ELl:TRÓLITOS NO PLASMA SEMINAI.}

As concentraçōes médias de Na, K, Ca e Mg, expressas em $\mathrm{mg} / 100 \mathrm{ml}$, estāo contidas na Tab.3. Os coeficientes de variaçāo entre as amostras foram baixos, oscilando entre $6 \%$, para o $\mathrm{Mg}$ e $30 \%$, para o $\mathrm{K}$.
\#** Folimetro de chama tit.l-mexdelo 100-Corning Brasil Leda. ****** Modelo 403-PERKIN - ELMER

******* Statistical Package for Soctal Science (SPSS - PC +), Chicago, SPSS Inc, . 1988 


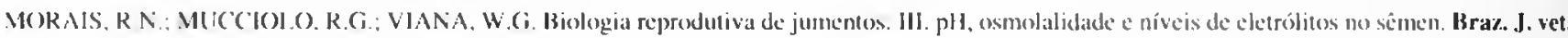
Kes. anim. Sci. Sáo Paulo, 1. 31, n.2. p. 14.5-5I, 1994.

\section{TABBIA I}

Média $(\bar{x})$ e desvio paldräo (DP) do pH docjaculado de jumentos. incluindo os valores máximon c mínimos. Säo Carlos - SP. $1988 / 89$.

\begin{tabular}{|c|c|c|c|c|c|}
\hline Jumezntos & $n$ & $\bar{x}$ & $\mathrm{DP}$ & Máximo & Mínimo \\
\hline$\Lambda$ & 14 & $7.26^{3}$ & 0.17 & 7.6 & 7,0 \\
\hline$B$ & 14 & $7.21^{\mathrm{a}}$ & 0.17 & 7.6 & 7.0 \\
\hline$C^{\prime}$ & 1.5 & $7.39^{\circ}$ & 0.22 & 7.8 & 7.0 \\
\hline I) & 06 & $7 . .36^{\circ}$ & 0.19 & 7.6 & 7.1 \\
\hline l: & II & $7 . .32^{a}$ & 0.11 & 7.6 & 7.2 \\
\hline F & 10 & $7.23^{\circ}$ & 0.13 & 7.4 & 7.0 \\
\hline Jonal & 70 & 7.29 & 0.18 & 7.8 & 7,0 \\
\hline
\end{tabular}

n - número de cjaculaddos

i = médias com diferentes tetras diferem signilicantemente $(\alpha,=0.05)$

TABFI.A 2

Média (x) a desvio padrão (DP) da osmolalidade (mOsm) do ejaculado do jumentos, incluindo os valores máximos e mínimos. São Carlos - SP. 1988 89.

\begin{tabular}{|c|c|c|c|c|c|}
\hline Jumentos & $n$ & $\bar{x}$ & I)P & Máximo & Mínimo \\
\hline$A$ & 14 & $299,21^{a}$ & 22.81 & 373 & 273 \\
\hline 13 & 14 & $30\left(0.93^{2}\right.$ & 17.57 & 3.57 & 277 \\
\hline C & 15 & $300,07^{a}$ & 8.16 & 315 & 287 \\
\hline () & 06 & $310.0\left(0^{a}\right.$ & 9.75 & 313 & 286 \\
\hline $1:$ & | 1 & $310.000^{\mathrm{a}}$ & 8.88 & 3.34 & 3() 1 \\
\hline $\mathrm{I}:$ & 09 & $30.5 .67^{a}$ & 1.3 .09 & 33.5 & 292 \\
\hline Total & 69 & 302,39 & 15.03 & 373 & 273 \\
\hline
\end{tabular}

$\mathrm{n}=$ número de cjaculados

a = médias com difirentes ketras diferem significantemente $(\alpha=0,0.5)$

\section{DISCUSSÃO E CONCIUUSOESS}

Pela análise dos resultados obtidos para o pH seminal. ohserva-se que existe grande semelhança tanto entre reprodutores como entre amostras de um mesmo animal, com coeficiente de variação de apenas $2 \%$. As amostras por nós analisadas tenderam à neutralidade, corroborando ohservaçōes prévias ${ }^{14}$. sendo yue nenhum dos ejaculados apresentou pll excessivamente alcalino, descartando-se assim a possibjlidade de contaminações bacterianas ${ }^{5,28}$. A laixa de variação do $\mathrm{pH}$ ficou entre 7.0 e 7.8 valores estes, próximos aos apresentados para garanhozes $18,23,24,26,25,31$. Comparativamente aos estudos realizados em jumentos, também existe bastante semelhançá entre os resultados $s^{6.8 .9} 910,11,19$, porém 0 menor valor registrido em nosso estudo foi 7,0, ficando acima dos limites inferiores anotados por outros pesquisadores ${ }^{4,9 .} 11$ No entanto. a média total obtida para os ejaculados analisados no presente estudo está incluida na faixa de variação proposta por CIIEVALIER'(1979) como sendo a ideal para o pH dos diluidores utilizados na conservação "in vilro" de sêmen equïino. Isto sugere que ambals as espécies possam ser tratadas da mesma mancirat ao se considerar o pH dos diluidores para conservação dos espermatozóides.

Com relação à determinação da osmolalidade, a leitura do ponto crioscópico provou ser uma técnica bastante simples e prática. Os resultados por nós obtidos foram relativamente constantes, com um coeficiente de variação da ordem de $5 \%$. Quando comparados com os valores reportados para garanhões ${ }^{18.24 .26 .27 .30 .3 .34}$ observou-se grande semelhança entro as duas espécies. Do mesmo modos. al média por nós registrada

TABRLA 3

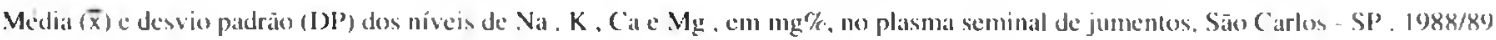

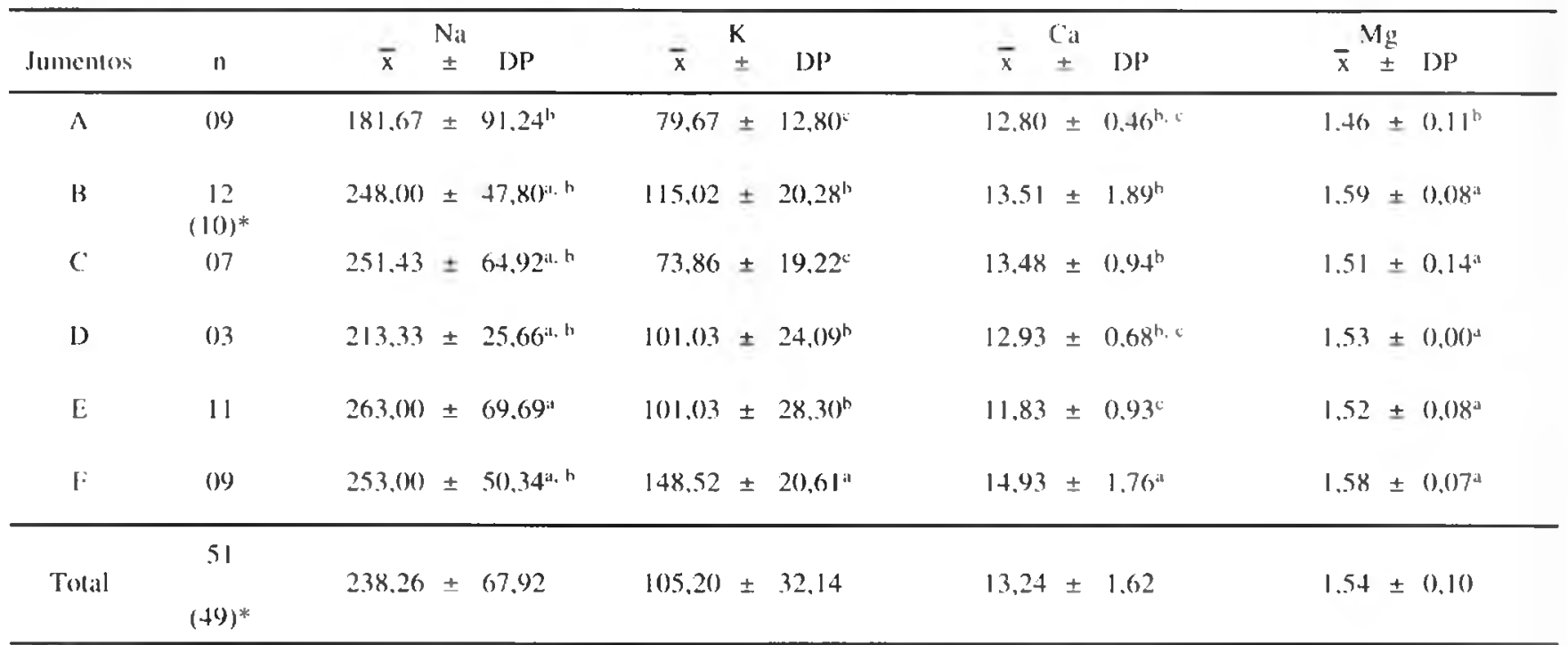

$n=$ número de ejaculados

a, b, $c=$ médias com diferentes letras diferem significantemente $(\alpha=0,05$,

* = número de ejaculados para as determinaçōes de Na 
MORAIS, R.N.; MUCCIOLO, R.G.; VIANA, W.G. Biologia reprodutiva de jumentos. III. pH, osmolalidade e níveis de eletrólitos no sêmen. Braz. J. vet. Res. anim. Sci., São Paulo, v. 31, n.2. p. 14.5-51, 1994.

foi praticamente a mesma conseguida por NISHIKAWA: WAIDE $^{19}$ (1951) em jumentos. As pequenas variações detectadas entre os nossos resultados cos de vários autores citados podem ser devidas, além das características individuais dos reprodutores estudados, às diferenças na metodologia utilizada. incluindo força e tempo de centrifugação das amostras de sêmen. Contudo, em função da grande semelhança entre os resultados obtidos para jumentos e garanhões, acreditamos que as considerações feitas anteriormente ${ }^{20.27 .28}$ para cqüinos, possam ser úteis na definição da osmolalidade ideal de diluidores a serem utilizados na conservação "in vitro" de sêmen asinino.

Analisando-se os níveis de eletrólitos detectados no plasma seminal, apresentados na Tab.3, verifica-se certa homogeneidade entre amostras com exceção apenas para as determinações de $\mathrm{Na}$ do jumento $\mathrm{A}$, que mostrou maior variabilidade. A presença de $\mathrm{Na}$ foi detectada nas amostras por nós analisadas numa concentração média de $238,26 \mathrm{mg} \%$, situando-se abaixo das médias reportadas para garanhões ${ }^{1}$. 12. 13. 21.34 e também para jumentos ${ }^{13}$. Provavelmente, esta diminuição na média global ocorreu em função das baixas concentrações observadas em três amostras do jumento $\mathrm{A}$. Coincidentemente ou não, corresponderam a três ejaculados que apresentaram gel. No entanto, não encontramos nenhuma referência a este fato na literatura consultada. Mesmo assim, numericamente não se pode definir uma diferença significante entre asininos e equiinos no que tange ao nivel de $\mathrm{Na}$ do plasma seminal.

Ao contrário do $\mathrm{Na}$, a concentração média de $\mathrm{K}$ por nós encontrada foi superior a praticamente todos os resultados conseguidos para garanhões ${ }^{1}$ 12. 21. 29, principalmente em relação aos dados de O'REILLY et al. ${ }^{21}$ (1979), cujos valores foram excessivamente baixos.

Para os níveis de Ca, as amostras foram bastante homogêneas e os resultados se encontram numa faixa intermediária entre as médias obtidas para eqüinos $21,29,34$, coincidindo com os valores de LANGOIS ${ }^{12}$ (1978) c AMANN et al. ' ( 1987). Já os valores de $\mathrm{Mg}$ registrados em nossas amostras estão bem abaixo das médias publicadas para garanhões ${ }^{16,21,29,34}$. Infelizmente, não temos outros dados relativos a jumentos para confrontarmos com os nossos, portanto, não se pode concluir que aqueles valores reflitam o normal para a raça ou espécie até que novas pesquisas o comprovem.

Do mesmo modo que a osmolalidade, as diferenças registradas entre os vários resultados analisados podem ser devidas a características espécic-específicas e a variações nos métodos utilizados nas análises. Não existe por exemplo, um método único de obtenção e armazenamento das amostras de plasma seminal, comum a todos os autores. No presente estudo adotamos uma força de centrifugação baixa, dentre os limites utilizados para a centrifugação do sêmen eqüino antes da congelação ${ }^{22}$.

A proporção média de $\mathrm{Na} / \mathrm{K}$ para as amostras por nós analisadas foi de 2,26 , ficando abaixo das proporções calculadas para os demais autores, as quais varia de 2,66 até 4,931,12,21,34. Isto nos leva a crer que, assim como para garanhões ${ }^{12}{ }^{15}$, os diluentes ricos em $\mathrm{Na}$ sejam prejudiciais aos espermatozóides de jumentos e que melhores resultados na congelação de sêmen "in vitro" possam ser obtidos quando mantidas proporções baixas de $\mathrm{Na} / \mathrm{K}$. Isto poderia simular, em escala muito menor, o que sucede a nível epididimário ${ }^{14}$. Obviamente, estas suposiçōes só poderão ser confirmadas em estudos posteriores, onde seja considerado também o comportamento metabólico dos espermatozóides de jumentos.

De modo geral, pela semelhança existente entre as características seminais de asininos e eqüinos, acredita-se que os métodos utilizados para congelação de sêmen de garanhōes possam, em princípio, ser utilizados ou não para o sêmen de jumentos. Estudos complementares poderão confirmar a viabilidade deste procedimento.

\section{SUMMARY}

Seminal samples of six jackass of Pêga breed were analysed for $\mathrm{pH}(\mathrm{n}=70)$, osmolality $(n=69)$ and Na $(n=49), K(n=\$ 1), C a$ $(n=51)$ and $\mathrm{Mg}(\mathrm{n}=51)$ levels determinations. The $\mathrm{pH}$ values ranged from 7.0 to 7.8 with the average of $7.29 \pm 1.18$ for the total ejaculate number. Seminal plasma osmolality stayed between 273 and $373 \mathrm{mOsm}$, with the total average of $302.89 \pm 15.03$ mOsm, which represents a freezing point depression of $0.56^{\circ} \mathrm{C}$. The meamconcentrations of $\mathrm{Na}, \mathrm{K}, \mathrm{Ca}$ and $\mathrm{Mg}$ in the seminal plasma were, respectively, $238.26 \pm 67.92,105.20 \pm 32.14,13.24 \pm 1.62$ and $1.54 \pm 0.10 \mathrm{mg} \%$. All the result obtained at the present study were compared to scientific information about donkeys and horses. Both species are quite semilar apart from $\mathrm{Mg}$ levels that was lower for the donkeys. Due to the similarities between jackass and stallion seminal characteristics we believe that procedures for equine semen "in vitro" conservation might also be used for jackass semen. Complementary research is need to confirm the procedures viability.

UNITERMS: Donkeys; Reproduction; Semen; pH; Osmotic pressure; Electrolytes 


\section{REFERÊNCIAS BIBI.IOGRÁFICAS}

01-A.MANN. R.P.: CRISTANELLI. M.J.; SQUIRES. E.L. Proteins in stallion seminal plasma.J. Reprod. Fertil., p.113-20, 1987. Supplement 35.

02-AMANN, R.P.; PICKETT, B.W. Principles ol cryopreservation and a review of cryopreservation of slallion spermatozoal. Equine Vet. Sci., v.7, n.3., p. 145-7.3, 1987.

0.3-BASSET, J.: IDFNNEY. R.C.; JEFFERY, (i.H.: MENIDHAM. J. Vogel - Análise inorgânica quantitativa. 4.ed. Rio de Janeiro. Cinanabara Dois, 1981

04-BIFI.ANSKI. W: WIERZBOWSKI, S. Some properties of output of semen of jackass. Acta Biol. Cracov. $S$. Zool., v.5. p.117-24, 1962.

05-CHEVALIER, F.C. Contribution a l'etude de l'insemination artificielle chez le cheval. Alfort, 1979. (Thése pour le doctorat Vélérinaire) - Fecole Nationale Vétérinaire D’Alfort.

06-CRAGLE.R.G.;SALISBURY.G.W. Factores inlluencing metabolic activity of bull spermatozoa. IV. pH, osmonic pressure, and the cations, sodium, potassium and calcium. J. Dairy Sci..v.42. p.1304-13. 1959.

07-GLATZLLL, P.; HL HOUSSAIN. K.; TIBARY. A. Pferdeund Esclhengste der marokkanischen Landespferd und amulticrzucht. Erste Lrgbnisse aus deim Sinsaty von Flussig - und Gefriersamen fur die Maulticrproduktion. Berl. Munch. Tierarztl. Wochenschr., v.94., p.445-9. 1981.

08-HENRY. M.; GASTAL. E.L.: MLIRA. C.; DIAZ. A.P.Caracleristicas do sêmen de jumentos da raça nordestina. In: CONGRESSO BRASILEIRO DE REPRODUÇĀO ANIMAL, 7.. Belo Iorizonte. 1987. Programa. Colégio Brasileiro de Reprodução Animal. 1987. p.72

(0)-KREUCHAUF, A. Zum Fortpflanzungs-gesschahem heim Esel (equus asinus). Munchen. 1983. (Inaugural Dissertation zur Erlangung der Tiermedizinischen Doktorwurde) - Tieraztlichen Fakultat der LudwigMaximilians. Universitat Munchen.

10-KREUCHAUF. A. Reproductive physiology in jackass. Anim. Res. Develop . 1.20, p.51-78, 1984.

II-KUIL.MI.V. I. Samenbiologische, biochemische und cryotechnische Entersuchungen and Eselsperma. Hannover. 1984. (Inaugural I)issertation Zur Erlangung der Tiermedisinischen Doktorwur(le) Tierazalichen Hoschule Hannover.

12-I.ANGOIS. P. Resumé de deux ammées d'élude sur l. sperme deatalon. Stocholm. 1978 apud
CHEVALIER $5.1979 . \mathrm{p}^{9}$

13-MANN. T.: MINOTAKIS, C.S.; POLGl, C. Semen composition and melabolism in the stallion and jackass. J. Reprod. Fertil.. v.5. p.109-22. 1963.

14-MANN. T. The biochemistry of semen and of the male reproductive tract. London. Butler \& Tamner, 1964

15-MARTIN, J.C : KI.LG. E.: GUNZEL. A. Centrifugation of stallion semen and its storage in large volume straws. J. Reprod. Fertil. p.47-51. 1979. Supplement 27.

16-MATOS. M.S.: VALE. W.G.: MEGALE, F. Algums constituintes bioquímicos do sêmen de eqüinos. Arq. Esc. Med. Vet. Univ. Fed. Bahia, v.2. p.29-36. 1977

17-NISHIKAWA, Y. Studies on the preservation of raw and frozen horse semen. J. Reprod. Ferlil.. p.99-104. 1975. Supplement 23

18-NISHIKAWA. Y: W:II)I:. Y. On artificial insemination in the horse. V. () properties of semen and the factors altecting them. Bull. Nat. Inst. Agric. Sci. Ser. (;., v.] p.13-28. 1951 apud Anim. Breed. Ibst., v.20. p.14. 1952. (R心)

19-NISHIKAWA. Y : WAIDF: Y'. Studies on reproduction in asses. VI. () n the mahoul of collection an properties of semen. Bull. Nat. Inst. Agric. Sci. Ser. G., v.1. p.3745. 1951 apud Anim. Breed. Abst.. v.20, p.14, 1952. (Resumo)

20-NISHIKAWA, Y.; WAIDE. Y.: SHINOMIYA. S. Studies on deep freezing of horse spermatomo. In: INTIERNATIONAL CONGIRESS ON ANIMAL RIPPRODUCTION AND ARTIIICIAI. INSI:MINATION.6. Paris. 1968. Proceedings. v.2. p.1589-91.

2I-O'RFILLY. P.J.: MCCORMACK. R.: O'MAHONY. K. The effects of collection procedures and freezing on concentration of some eletrolytes and glutamic oxaloacetic transaminase on stallion seminal plasma. Irish Vet. J.. v.33, n.4. p.55-9. 1979.

22-PAPA. F.O. Contribuição ao estudo de sêmen congelado de eqüinos: modificações metodológicas para o congelamento e inseminação artificial. Botucatu. 1987. Tese (livre Docência) - Faculdade de Medicina Veterinária e Zootecnia. Universidade Estadual Paulistat.

23-PATTIE, W.A.; DOWSETT, K.F. The repeatability of seminal characteristics of stallations.J. Reprod. Fertil. p.9-13. 1982. Supplement 32.

24-PICKETT. B.W.; FAULKNER, L.C.: SHUTERLAND. T.M. Effect of month and stallion on seminal 
characteristics and sexual behavior. J. Anim. Sci., v.31, p.713-28, 1970.

5-PICKETT, B.W.; SQUIRES, E.L.; MCKINNON, A.O. Procedures for collection and utilization of stallion semen for artificial insemination. Fort Collins, Anim. Reprod. Lab., Colorado State University, 1987.

6-PICKETT, B.W.; SULLIVAN, J.J.; SEIDEL JUNIOR, G.E. Reproductive physiology of the stallion. V. Effect of frequency of ejaculation on seminal characteristics and spermatozoal output. J. Anim. Sci., v.40, p.917$23,1975$.

7-RAJAMANNAN, A.H.J.; ZEMJANIS, R.; ELLERY, J. Freezing and fertility studies with stallion semen. In: INTERNATIONAL CONGRESS ON ANIMAL REPRODUCTION AND ARTIFICIAL INSEMINATION. Paris, 1968. Proceedings. v.2, p.1601-4.

8-SALISBURY, G.W.; VANDEMARK, N.L. Fisiologia de la reprodución e Inseminación Artificial de los bóvidos. Zaragoza, Acríbia, 1964.

9-SATO, K.; MIYAKE, M.; SUGAWARA, M.; TAKEYAMA, T.; COHASHI, S.; IRUAMA, N.; SUGYRAMA, K.; IOVE, H.; YASHIKAWA, T. Multivariate analysis of semen characters in 3-year- colts and stallions in the breeding and non breeding seasons. Jap. J. Zootech. Sci., v.44, p.476-82, 1973.

30-SLOVTZOV, B.1916 apud MANN ${ }^{14}$, 1964. p.108.

31-SQUIRES, E.L.; AMANN, R.P.; PICKETT, B.W.; BERNDTSON, W.E.; SHIDELER, R.K.; VOSS, J.L. Effect of fenbendazole on reproductive funcion in stallions. Theriogenology, v.9, p.447-55, 1978

32-VARNER, D.D.; BLANCHARD, T.L.; LOVE, C.L.; GARCIA, M.C.; KENNEY, R.M. Effects of semen fractionation and dilution ratio on equine spermatozoal motility parameters. Theriogenology. v.28, p.70923,1987

33-WEISMAN, N.; LEGGI, V.J.P. Inorganic ions. Osmolality. In: HENRY, R.J.; CANNON, D.C.; WINKELMAN, J.W. eds.Clinical chemistry, principles and technics. 2.ed. New York, Harper \& Row, 1974. p.736-9.

34-YAMANE, J., 1920 apud MANN ${ }^{14}$, 1964. p.108.

Recebido para publicação em 12/03/93 Aprovado para publicação em 19/10/93 